\title{
Effects of neutrino inverse seesaw mechanism on the sparticle spectrum in CMSSM and NUHM2
}

\author{
I. Gogoladze, ${ }^{a}$ B. He, ${ }^{a}$ A. Mustafayev, ${ }^{b}$ S. Raza ${ }^{c}$ and Q. Shafi ${ }^{a}$ \\ ${ }^{a}$ Bartol Research Institute, Department of Physics and Astronomy, University of Delaware, \\ Newark, DE 19716, U.S.A. \\ ${ }^{b}$ Department of Physics and Astronomy, University of Hawaii, \\ Honolulu, HI 96822, U.S.A. \\ ${ }^{c}$ State Key Laboratory of Theoretical Physics \\ and Kavli Institute for Theoretical Physics China (KITPC), \\ Institute of Theoretical Physics, Chinese Academy of Sciences, \\ Beijing 100190, P.R. China \\ E-mail: ilia@bartol.udel.edu, hebin@udel.edu, azar@phys.hawaii.edu, \\ shabbar@itp.ac.cn, shafi@bartol.udel.edu
}

ABSTRACT: We study the implications of the inverse seesaw mechanism (ISS) on the sparticle spectrum in the Constrained Minimal Supersymmetric Standard Model (CMSSM) and Non-Universal Higgs Model (NUHM2). Employing the maximal value of the Dirac Yukawa coupling involving the up type Higgs doublet provides a $2-3 \mathrm{GeV}$ enhancement of the lightest CP-even Higgs boson mass. This effect permits one to have lighter colored sparticles in the CMSSM and NUHM2 scenarios with LSP neutralino, which can be tested at LHC14. We present a variety of LHC testable benchmark points with the desired LSP neutralino dark matter relic abundance.

KeYWORDS: Supersymmetry Phenomenology

ArXiv ePrint: 1401.8251 


\section{Contents}

1 Introduction 1

2 Inverse seesaw mechanism and Higgs boson mass 2

3 Phenomenological constraints and scanning procedure 4

4 Results 5

4.1 CMSSM and inverse seesaw 5

4.2 NUHM2 and inverse seesaw 11

5 Conclusions 14

\section{Introduction}

The recent discovery of the Standard Model (SM)-like Higgs boson with mass $m_{h}=$ $125.5 \pm 0.5 \mathrm{GeV}$ by the ATLAS [1] and CMS [2] experiments at the Large Hadron Collider (LHC) has sparked detailed examinations of viable regions of the parameter space of low scale supersymmetry. This is largely motivated by the fact that the Minimal Supersymmetric Standard Model (MSSM) predicts an upper bound on the mass of the lightest CP-even Higgs boson mass, $m_{h} \lesssim 135 \mathrm{GeV}$ [3]. The Higgs boson mass and the corresponding sparticle spectrum strongly depend on the soft supersymmetry breaking (SSB) parameters [4], which can be tested at the LHC (see, for instance [5-20]).

In low scale supersymmetry, a Higgs boson mass of around $125 \mathrm{GeV}$ requires either a relatively large value, $\mathcal{O}$ (few-10) $\mathrm{TeV}$, for the geometric mean of top squark masses [10], or a large SSB trilinear $A_{t}$-term, with a geometric mean of the top squark masses of around a $\mathrm{TeV}$ [11-20]. The presence of heavy top squarks typically yields a heavy sparticle spectrum in gravity mediated supersymmetry breaking [21-25], if universality at $M_{\mathrm{GUT}}$ of sfermion masses is assumed. It is especially hard in this case to achieve colored sparticles lighter than $2.5 \mathrm{TeV}$.

The current LHC lower bounds on the colored sparticle masses from LHC data are $m_{\tilde{g}} \gtrsim 1.5 \mathrm{TeV}$ (for $m_{\tilde{g}} \sim m_{\tilde{q}}$ ), and $m_{\tilde{g}} \gtrsim 0.9 \mathrm{TeV}$ (for $m_{\tilde{g}} \ll m_{\tilde{q}}$ ) [26, 27], and it is expected that the LHC14 can test squarks and gluinos with masses up to $3.5 \mathrm{TeV}$ [28]. In order to be able to reduce the sparticle masses to more accessible values in models with universal sfermion and gaugino masses, we require additional contributions from new physics, which preserves gauge coupling unification.

Solar and atmospheric neutrino oscillation experiments have established that at least two neutrino states are massive [29]. On the theoretical side the nature of the physics responsible for neutrino masses and flavor properties remains largely unknown and is a 
subject of extensive investigations [30]. Since our goal is to lower the sparticle mass spectrum while preserving gauge coupling unification, we utilize in this paper the inverse seesaw mechanism (ISS) for generating the light neutrino masses [31, 32]. Introducing only SM singlet fields allows one to realize the ISS mechanism, and all new fields can be below the $\mathrm{TeV}$ scale. In addition, we can have $\mathcal{O}(1)$ Dirac Yukawa couplings involving the up type Higgs doublet. It has been shown in refs. [33-35] that the Dirac Yukawa coupling can impact the lightest CP-even Higgs boson mass through radiative corrections and increase it by $2-3 \mathrm{GeV}$ when the additional new fields are SM singlets. The ISS mechanism can also be realized using $\mathrm{SU}(2)_{W}$ weak triplets [33, 34], and in this case the Higgs mass can be enhanced by more than $10 \mathrm{GeV}$.

In this paper we restrict ourselves to the case of SM singlet fields since we do not want to disturb gauge coupling unification. An enhancement by $2-3 \mathrm{GeV}$ of the CP-even SM-like Higgs boson mass, as we will show, can yield significant reductions of sparticle masses in the Constrained Minimal Supersymmetric Standard Model (CMSSM) [21-25] and NonUniversal Higgs Model with $m_{H_{u}}^{2} \neq m_{H_{d}}^{2}$ (NUHM2) [36-38]. Here $m_{H_{u}}^{2}$ and $m_{H_{d}}^{2}$ denote the SSB mass square terms for the up and down type MSSM Higgs doublets respectively.

The outline for the rest of the paper is as follows. In section 2 we briefly describe the model including the SSB parameters, the range of values employed in our scan, and the scanning procedure. The relevant experimental constraints that we have employed are described in section 3. The results pertaining to CMSSM, CMSSM-ISS and NUHM2-ISS are discussed in section 4, and our conclusions are summarized in section 5.

\section{Inverse seesaw mechanism and Higgs boson mass}

In order to explain non-zero neutrino masses and mixings by the ISS mechanism [31, 32], we supplement the MSSM field content with three pairs of MSSM singlet chiral superfields $\left(N_{i}^{c}+N_{i}\right), i=1,2,3$, and a singlet chiral superfield $S$ which develops a vacuum expectation value (VEV) comparable to or less than the electroweak scale. The part of the renormalizable superpotential involving only the new chiral superfields is given by

$$
W \supset Y_{N_{i j}} N_{i}^{c} H_{u} L_{j}+\lambda_{N_{i j}} S N_{i} N_{j}+m_{i j} N_{i}^{c} N_{j}
$$

Here $Y_{N_{i j}}$ and $\lambda_{N_{i j}}$ are dimensionless couplings and $m_{i j}$ is a mass term. A non-zero VEV for the scalar component of $S$ generates the lepton-number-violating term $\mu_{s} N_{i} N_{j} \equiv$ $\lambda_{N_{i j}}\langle S\rangle N_{i} N_{j}$ and, as a result, Majorana masses for the observed neutrinos can be generated. The coupling $\lambda_{N_{i j}} S N_{i} N_{j}$ is preferred over the direct mass term $\mu N_{i} N_{j}$, with the former yielding the desired mass terms for the $N$ fields with a non-zero $\langle S\rangle$. A singlet chiral superfield $S$ can make it easier to find extension of the SM gauge group with help from a suitable symmetry (see, for instance, refs. [33, 34, 39-47]), and avoid terms which otherwise may spoil the ISS mechanism.

The SSB terms pertaining to the fields $N_{i}^{c}$ and $N_{i}$ are given by

$$
\mathcal{L}^{\text {soft }} \supset m_{N^{c}}^{2}{\widetilde{N^{c}}}^{\dagger} \widetilde{N^{c}}+m_{N}^{2} \widetilde{N}^{\dagger} \widetilde{N}+\left[A_{\nu}^{i j} \widetilde{L}_{i} \widetilde{N}^{c}{ }_{j} H_{u}+B_{m}^{j k}{\widetilde{N^{c}}}_{j} \widetilde{N}_{k}+B_{\mu_{N}}^{j k} \widetilde{N}_{j} \widetilde{N}_{k}+\text { h.c. }\right] \text {, }
$$




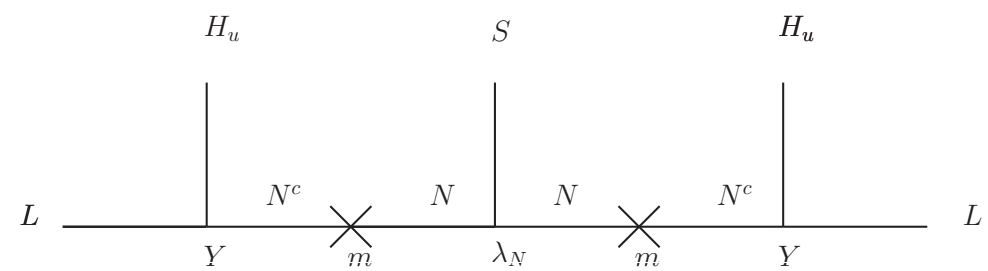

Figure 1. Supergraph leading to dimension six operator for neutrino masses.

where the SSB parameters are prescribed at the TeV SUSY breaking scale. In the ISS case there are regions of the SSB parameter space for which one of the sneutrinos can be the lightest supersymmetric particle (LSP). The phenomenology of models of this kind has been studied in refs. [39-47]. In our present work we assume that the lightest neutralino is the LSP, and a spectrum of this nature can be realized both in the CMSSM and NUHM2 if we assume that all sfermions, including the $N_{i}^{c}$ and $N_{i}$ fields, have universal SSB mass terms at $M_{\mathrm{GUT}}$.

According to the superpotential in eq. (2.1), after integrating out the $\left(N_{i}^{c}+N_{i}\right)$ fields, the neutrino mass arises from the effective dimension six operator (figure 1):

$$
\frac{L L H_{u} H_{u} S}{M_{6}^{2}} .
$$

We assume here that $M_{6} \delta_{i j} \equiv m_{i j}$ is larger than the electroweak scale. Also, in eq. (2.3) the family and $\mathrm{SU}(2)_{W}$ gauge indices are omitted.

Following the electroweak symmetry breaking, the neutrino Majorana mass matrix is generated:

$$
m_{\nu}=\frac{\left(Y_{N}^{T} Y_{N}\right) v_{u}^{2}}{M_{6}} \times \frac{\lambda_{N}\langle S\rangle}{M_{6}} .
$$

For simplicity, we set $Y_{N} \equiv Y_{N_{i j}}$ and $\lambda_{N} \equiv \lambda_{N_{i j}}$, and $v_{u},\langle S\rangle$ are the VEVs of $H_{u}$, and the $S$ field. Eq. (2.4) implies that even if we require $Y_{N} \sim \mathcal{O}(1)$ and $M_{6} \sim 1 \mathrm{TeV}$, the correct mass scale for the light neutrinos can be reproduced by suitably adjusting $\lambda_{N}\langle S\rangle$.

Keeping $Y_{N} \sim \mathcal{O}(1)$ will provide sizable contribution to the lightest CP-even Higgs mass, which is given by [48, 49]

$$
\left[m_{h}^{2}\right]_{N}=n \times\left[-M_{Z}^{2} \cos ^{2} 2 \beta\left(\frac{1}{8 \pi^{2}} Y_{N}^{2} t_{N}\right)+\frac{1}{4 \pi^{2}} Y_{N}^{4} v^{2} \sin ^{4} \beta\left(\frac{1}{2} \widetilde{X}_{Y_{N}}+t_{N}\right)\right],
$$

where

$$
t_{N}=\log \left(\frac{M_{S}^{2}+M_{6}^{2}}{M_{6}^{2}}\right), \quad \widetilde{X}_{Y_{N}}=\frac{4 \widetilde{A}_{Y_{N}}^{2}\left(3 M_{S}^{2}+2 M_{6}^{2}\right)-\widetilde{A}_{Y_{N}}^{4}-8 M_{S}^{2} M_{6}^{2}-10 M_{S}^{4}}{6\left(M_{S}^{2}+M_{6}^{2}\right)^{2}},
$$

and

$$
\widetilde{A}_{Y_{N}}=A_{Y_{N}}-Y_{N}\langle S\rangle \cot \beta
$$

Also, $A_{Y_{N}} \equiv A_{\nu}^{i j}$ is the SSB mixing parameter in eq. (2.2), $n$ is the number of pairs of new MSSM singlets, $M_{S}=\sqrt{m_{\tilde{t}_{L}} m_{\tilde{t}_{R}}}$ defines the SUSY scale, and $v=174.1 \mathrm{GeV}$ is the electroweak VEV. 
We incorporate the ISS mechanism in CMSSM and NUHM2 and scan the SUSY parameter space using the ISAJET 7.84 package [50]. We modify the code by including the additional contributions from eq. (2.5) to the lightest CP-even Higgs boson mass.

\section{Phenomenological constraints and scanning procedure}

We employ the ISAJET 7.84 package [50] to generate sparticle spectrum over the fundamental parameter space. In this package, the weak scale values of the gauge, third generation Yukawa couplings, including the Yukawa coupling $N_{i}^{c} H_{u} L_{j}$ from ISS, are evolved to $M_{\mathrm{GUT}}$ via the MSSM renormalization group equations (RGEs) in the $\overline{D R}$ regularization scheme. With the boundary conditions given at $M_{\mathrm{GUT}}$, all of the SSB parameters, along with the gauge and Yukawa couplings, are evolved back to the weak scale $M_{\mathrm{Z}}$. The data points collected all satisfy the requirement of radiative electroweak symmetry breaking condition with the neutralino in each case being the LSP.

We have performed Markov-chain Monte Carlo (MCMC) scans for the following CMSSM parameter range:

$$
\begin{aligned}
0 \leq m_{0} & \leq 10 \mathrm{TeV} \\
0 \leq m_{1 / 2} & \leq 5 \mathrm{TeV} \\
-3 \leq A_{0} / m_{0} & \leq 3 \\
3 \leq \tan \beta & \leq 60
\end{aligned}
$$

with $\mu>0$ and $m_{t}=173.3 \mathrm{GeV}$ [51]. We use $m_{b}^{\overline{D R}}\left(M_{\mathrm{Z}}\right)=2.83 \mathrm{GeV}$ which is hard-coded into ISAJET. Here $m_{0}$ is the universal SSB mass parameter for MSSM sfermions, Higgs and additional $N^{c}, N$ and $S$ fields, $m_{1 / 2}$ is the gaugino mass parameter, $\tan \beta$ is the ratio of the VEVs of the two MSSM Higgs doublets, and $A_{0}$ is the MSSM universal SSB trilinear scalar coupling. In order to maximize the contribution from the ISS mechanism to the Higgs boson mass, we set $\tilde{X}_{Y_{N}}=4$, following refs. [33, 34].

In the case of NUHM2, in addition to the above mentioned parameters we have two additional independent mass parameters $m_{H_{d}}$ and $m_{H_{u}}$. We use the following parameter range for them:

$$
\begin{aligned}
& 0 \leq m_{H_{u}} \leq 10 \mathrm{TeV} \\
& 0 \leq m_{H_{d}} \leq 10 \mathrm{TeV}
\end{aligned}
$$

To maximize the impact of ISS on the sparticle spectrum, we set $\lambda_{N}=0.7$. This is the maximal value of $\lambda_{N}$ at low scale that remains perturbative up to $M_{\mathrm{GUT}}$. We also assume that $M_{6}$ is larger than $M_{S}$, in order that the neutralino rather than sneutrino is the LSP.

After collecting the data, we impose the mass bounds on all the particles [52] and use the IsaTools package $[53,54]$ and ref. [55] to implement the following phenomenological 
constraints:

$$
\begin{aligned}
m_{h} & =123-127 \mathrm{GeV} & & {[1,2,56] } \\
0.8 \times 10^{-9} \leq \mathrm{BR}\left(B_{s} \rightarrow \mu^{+} \mu^{-}\right) & \leq 6.2 \times 10^{-9}(2 \sigma) & & {[57] } \\
2.99 \times 10^{-4} \leq \mathrm{BR}(b \rightarrow s \gamma) & \leq 3.87 \times 10^{-4}(2 \sigma) & & {[58] } \\
0.15 \leq \frac{\mathrm{BR}\left(B_{u} \rightarrow \tau \nu_{\tau}\right)_{\mathrm{MSSM}}}{\operatorname{BR}\left(B_{u} \rightarrow \tau \nu_{\tau}\right)_{\mathrm{SM}}} & \leq 2.41(3 \sigma) & & {[59] . }
\end{aligned}
$$

As far as the muon anomalous magnetic moment $a_{\mu}$ is concerned, we require that the benchmark points are at least as consistent with the data as the SM.

For the benchmark points presented in table 1 and 2, we require that the neutralino LSP relic abundance lies in the interval $0.0913 \leq \Omega_{\mathrm{CDM}} h^{2} \leq 0.1363$ measured by WMAP for the Cold Dark Matter (CDM) abundance [60].

Finally we implement the following following bounds on the sparticle masses:

$$
m_{\tilde{g}} \gtrsim 1.5 \mathrm{TeV}\left(\text { for } m_{\tilde{g}} \sim m_{\tilde{q}}\right) \quad \text { and } \quad m_{\tilde{g}} \gtrsim 0.9 \mathrm{TeV}\left(\text { for } m_{\tilde{g}} \ll m_{\tilde{q}}\right) \quad[26,27] \text {. }
$$

\section{Results}

\subsection{CMSSM and inverse seesaw}

In this section we present our results for the CMSSM and the CMSSM with additional ISS contribution (CMSSM-ISS). The main idea behind the presentation of these results is to show that these two scenarios have quite distinct features as far as choice for the fundamental parameters of the models is concerned. In figure 2, the left panels represent our results for the CMSSM, while the right panels display our results for the CMSSM-ISS. Here grey points satisfy REWSB and the LSP neutralino requirement. The orange points represent solutions which satisfy the mass bounds and B-physics bounds from section 3 . Solutions in blue color are a subset of orange points and satisfy the requirement $123 \mathrm{GeV} \lesssim$ $m_{h} \lesssim 127 \mathrm{GeV}$. We display our results for a wider range of neutralino LSP relic abundance, $\Omega h^{2}$, keeping in mind that one can always find points within the WMAP range with dedicated scans within the orange regions.

For instance, the graph in $m_{0}-m_{1 / 2}$ plane shows that for the CMSSM case, the Higgs mass bounds excludes simultaneously small values for $m_{0}$ and $m_{1 / 2}$, while in the CMSSMISS case, we can have relatively small values for $m_{1 / 2}(<800 \mathrm{GeV})$ and $m_{0}(<400 \mathrm{GeV})$, consistent with all constraints given in section 3 . There is also noticeable difference between CMSSM and CMSSM-ISS in the $A_{0} / m_{0}-m_{0}$ plane. In the CMSSM case, for instance, we find the minimal values $m_{0} \sim 700 \mathrm{GeV}$ for $A_{0} / m_{0}=-3$, and for $A_{0} / m_{0}=3$ we have $m_{0} \sim 1.3 \mathrm{TeV}$. In CMSSM-ISS, on the other hand, the corresponding minimum $m_{0}$ values vary from $400 \mathrm{GeV}$ to $1.1 \mathrm{TeV}$. In the $m_{0}$-tan $\beta$ plane too, considering the blue points, we see in the left panel that for a minimum value $m_{0} \sim 700 \mathrm{GeV}$, the corresponding $\tan \beta$ value is around 16. In the right panel, on the other hand, $\tan \beta$ is again around 16 but now the minimum value of $m_{0}$ is $\sim 300 \mathrm{GeV}$.

In figure 3 we show plots of $m_{0}$ versus $\mu$. The color coding is the same as in figure 2 with the left and right panels representing CMSSM and CMSSM-ISS respectively. This 

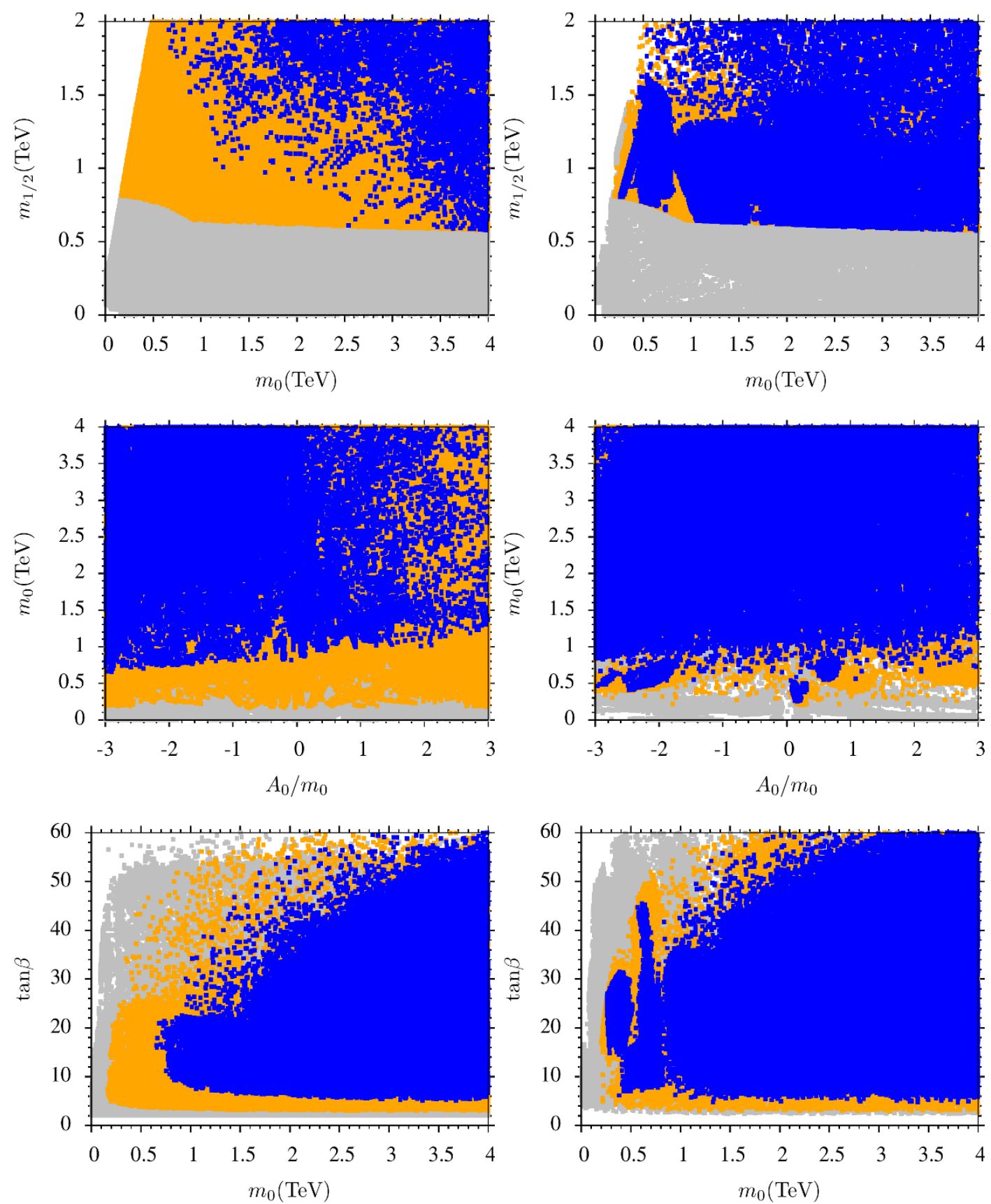

Figure 2. Plots in $m_{0}-m_{1 / 2}, A_{0} / m_{0}-m_{0}$ and $m_{0}$-tan $\beta$ planes for CMSSM (left panel) and CMSSMISS (right panel). Grey points satisfy REWSB and LSP neutralino conditions. Orange point solutions satisfy mass bounds and B-physics bounds given in section 3. Points in blue are a subset of orange points that satisfy the Higgs mass bound, $123 \mathrm{GeV} \lesssim m_{h} \lesssim 127 \mathrm{GeV}$.

figure shows very distinct features of the two scenarios. Considering the orange points, in CMSSM-ISS we have solutions with $\mu \gtrsim 1 \mathrm{TeV}$, in contrast with the CMSSM, where we have solutions with small, as well as large values of $\mu$. The reason for this difference is that in CMSSM-ISS, $m_{H_{u}}^{2}$ gets new contribution from the loop induced by the coupling $N_{i}^{c} H_{u} L_{j}$ 

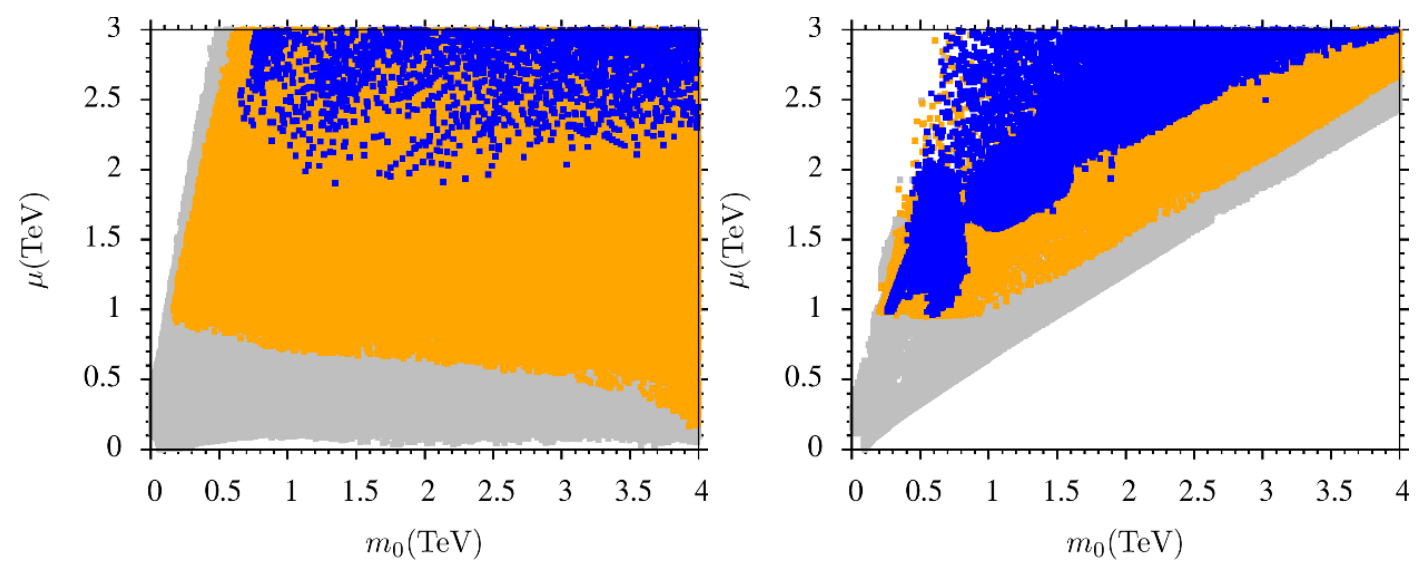

Figure 3. Plots in $m_{0}-\mu$ plane for CMSSM (left panel) and CMSSM-ISS (right panel). Color coding is the same as in figure 2 .

in addition to the top quark loop, which makes $\mu$ relatively heavy. Thus, in the CMSSMISS case we do not have the so-called focus point/hyperbolic branch scenario [61-64] while it is still a viable solution in the CMSSM case.

In figure 4 , we show graphs in $m_{\tilde{\chi}_{1}^{0}-} m_{\tilde{t}_{1}}$ and $m_{\tilde{\chi}_{1}^{0}}-m_{\tilde{\tau}_{1}}$ planes. The color coding is the same as in figure 2, except that the solutions in red are a subset of solutions in blue and also satisfy the relic abundance bound $0.001 \leq \Omega h^{2} \leq 1$. These graphs show that despite the fact that there are differences in the space of fundamental parameters, the mass spectrum for $\chi_{1}^{0}, \tilde{t}_{1}$ and $\tilde{\tau}_{1}$ turn out to be more or less identical.

For instance, in the $m_{\tilde{\chi}_{1}^{0}}-m_{\tilde{t}_{1}}$ plane we see that we have NLSP $\tilde{t}_{1}$ in the mass range of $\sim 260-500 \mathrm{GeV}$ in both cases. Similar results were also reported in $[65,66]$ in the case of $b-\tau$ Yukawa coupling unification in CMSSM and SU(5). It was shown in $[67,68]$ that the region of parameter space with stop-neutralino mass difference of $20 \%$ is ruled out for $m_{\tilde{t}_{1}} \lesssim 140 \mathrm{GeV}$. In the $m_{\tilde{\chi}_{1}^{0}-} m_{\tilde{\tau}_{1}}$ plane, we note that NLSP $\tilde{\tau}_{1}$ has the same mass range in CMSSM and CMSSM-ISS. The reason why we have comparable intervals for $m_{\tilde{t}_{1}}$ and $m_{\tilde{\tau}_{1}}$ in CMSSM and CMSSM-ISS is that low values for both sparticle masses are acheived via fine tuning involving the trilinear SSB terms, while the addition of ISS to CMSSM mostly affects the first two generation sparticle masses.

In figure 5 , we present graphs in $m_{\tilde{\chi}_{1}^{0}-m_{A}}$ and $m_{\tilde{\chi}_{1}^{0}-m_{\tilde{\chi}_{1}^{ \pm}}}$planes, with color coding the same as in figure 4 . The graphs in $m_{\tilde{\chi}_{1}^{0}-m_{A}}$ plane show that we do not have the $A$-resonance solution [69-74], and the reason can be understood from the following equation:

$$
m_{A}^{2}=2|\mu|^{2}+m_{H_{u}}^{2}+m_{H_{d}}^{2} .
$$

In CMSSM, since we have universal scalar masses and we require $m_{h} \sim 123-127 \mathrm{GeV}$, $m_{H_{u}}^{2}$ and $m_{H_{d}}^{2}$ are both large, and, as a result, $m_{A}$ is also large. This can be seen in the $m_{\tilde{\chi}_{1}^{0}-m_{A}}$ graph in the left panel. The solid black line in the graph represents the condition $2 m_{\tilde{\chi}_{1}^{0}}=m_{A}$ for the $A$-resonance solution [69-74].

We note that the solutions in orange color lie around the solid black line, but if we apply the constraint $123 \mathrm{GeV} \lesssim m_{h} \lesssim 127 \mathrm{GeV}$, the relevant blue points lie further from 

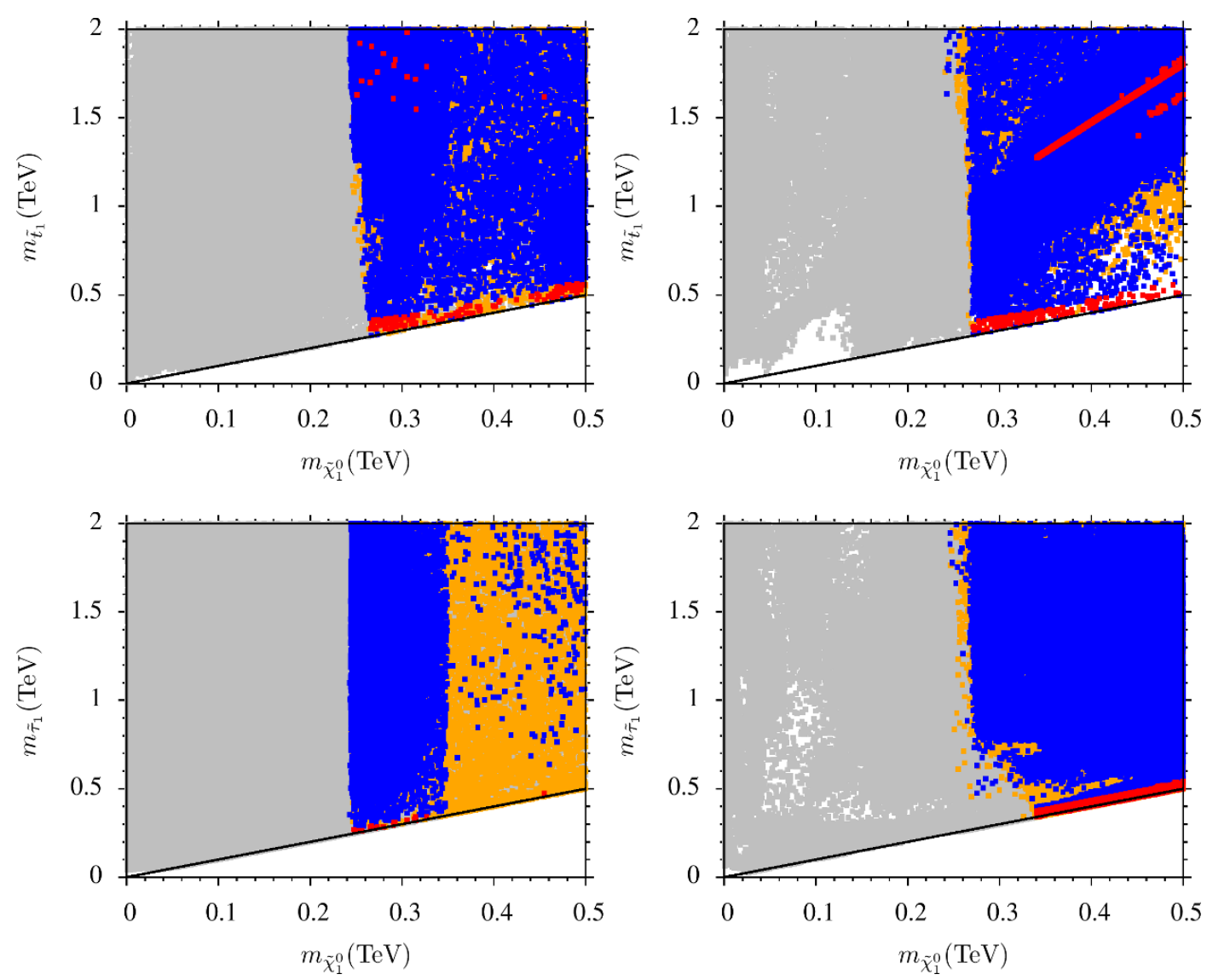

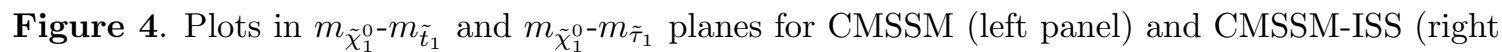
panel). The color coding is the same as in figure 2 except that red points are a subset of blue point solutions and also satisfy bounds for neutralino relic abundance, $0.001 \leq \Omega h^{2} \leq 1$.

the black line. In the right panel, which represents the CMSSM-ISS case, we note that both the orange and blue points are further away from the solid black line. This is because of two reasons. Firstly, as stated earlier, $\mu$ is larger because of extra contributions from the $N_{i}^{c} H_{u} L_{j}$ Yukawa coupling, and so the orange points move away from the solid black line. Secondly, as explained, in the CMSSM case the $m_{h}$ constraint makes solutions move away from the solid black line as $m_{A}$ becomes larger.

A more distinctive figure concerning the sparticle spectra in CMSSM and CMSSMISS is presented in the $m_{\tilde{\chi}_{1}^{0}-} m_{\tilde{\chi}_{1}^{ \pm}}$plane. In contrast to CMSSM (left panel), the figure for CMSSM-ISS is quite different. This is due to the fact that in CMSSM-ISS, the LSP neutralino is mostly a bino and the chargino mostly wino. Therefore, the ratio $m_{\tilde{\chi}_{1}^{0}} / m_{\tilde{\chi}_{1}^{ \pm}}$ is close to the ratio of $\mathrm{U}(1)$ and $\mathrm{SU}(2)$ gauge couplings, $g_{1} / g_{2} \approx 1 / 2$, and the points form a narrow strip.

In figure 6 we show $m_{\tilde{q}}$ versus $m_{\tilde{g}}$ for CMSSM (left panel) and CMSSM-ISS (right panel). The color coding is the same as in figure 2, except that the orange points do not include mass bounds for gluinos and the first two generation squarks. Dashed vertical and horizontal lines represent current squark and gluino mass bounds. We note that especially 

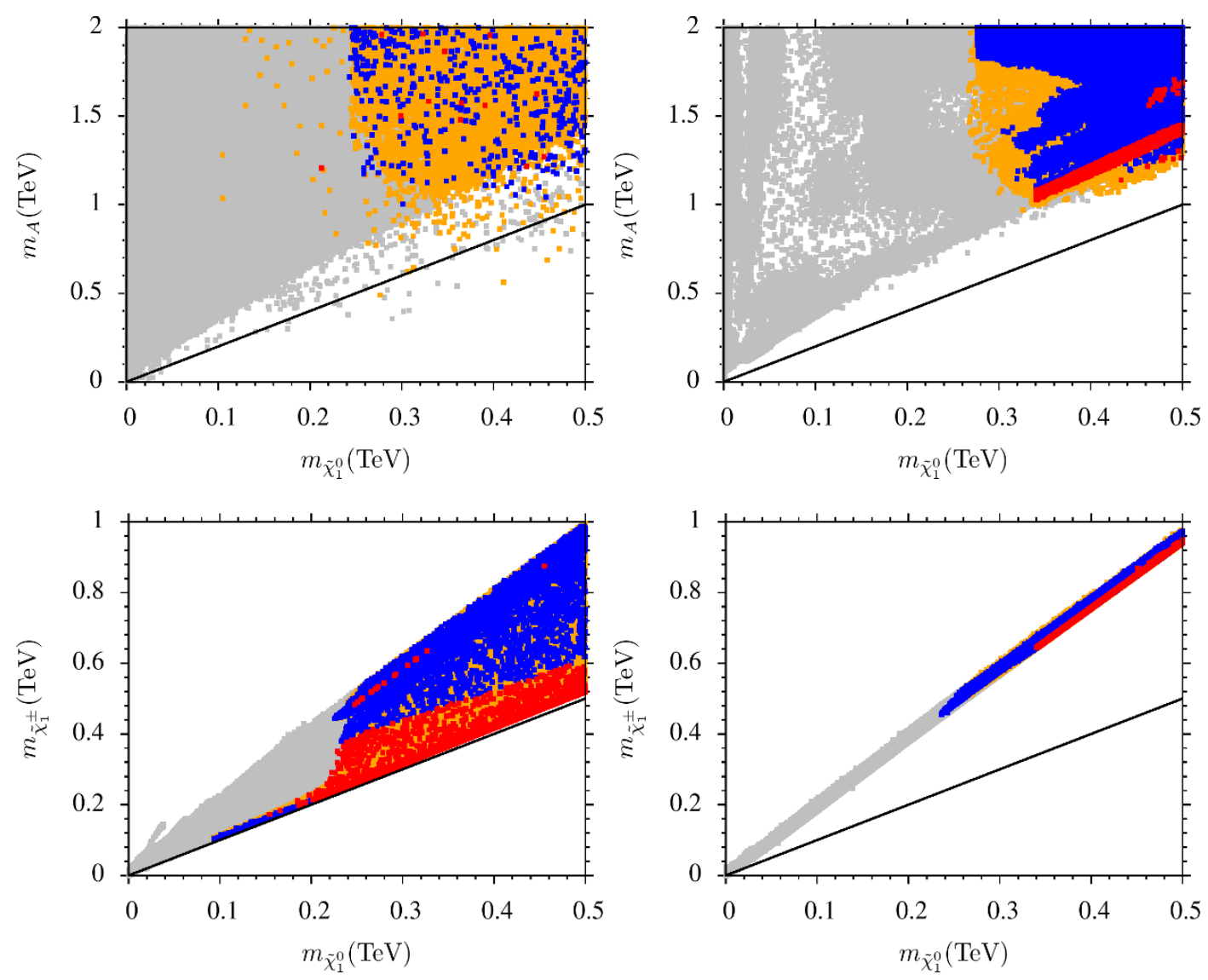

Figure 5. Plots in $m_{\tilde{\chi}_{1}^{0}}-m_{A}$ and $m_{\tilde{\chi}_{1}^{0}}-m_{\tilde{\chi}_{1}^{ \pm}}$planes for CMSSM (left panel) and CMSSM-ISS (right panel). The color coding is the same as in figure 4.

in the CMSSM the gluino mass bound excludes a significant portion of the parameter space which otherwise is consistent with the experimental data. The location of blue points relative to the orange points shows how the lower bounds on the squark and gluino masses are pushed up by $m_{h}$. It is interesting to observe that there are no red points with neutralino LSP dark matter within the reach of LHC14. Comparing results from $m_{\tilde{g}}-m_{\tilde{q}}$ panel with the results from figures 4 and 5, we conclude that in the CMSSM, the solution which yields the correct dark matter relic abundance predicts gluino and squarks masses that lie beyond the reach of the LHC14 [28].

On the other hand, comparison of left and right panels in figure 6 shows the impact of the ISS mechanism on the sparticle masses. We can see from the $m_{\tilde{g}}-m_{\tilde{q}}$ plot in the right panel that plenty of blue points are left after we apply the Higgs mass constraint $123 \mathrm{GeV} \lesssim m_{h} \lesssim 127 \mathrm{GeV}$. This means that in the presence of the ISS mechanism, most points satisfying all experimental constraints lie in the Higgs mass range $123 \mathrm{GeV} \lesssim m_{h} \lesssim$ $127 \mathrm{GeV}$, which is very different from the CMSSM case. There are also red points in the right panel which shows that we can have LHC testable solutions with the correct relic abundance of dark matter. 

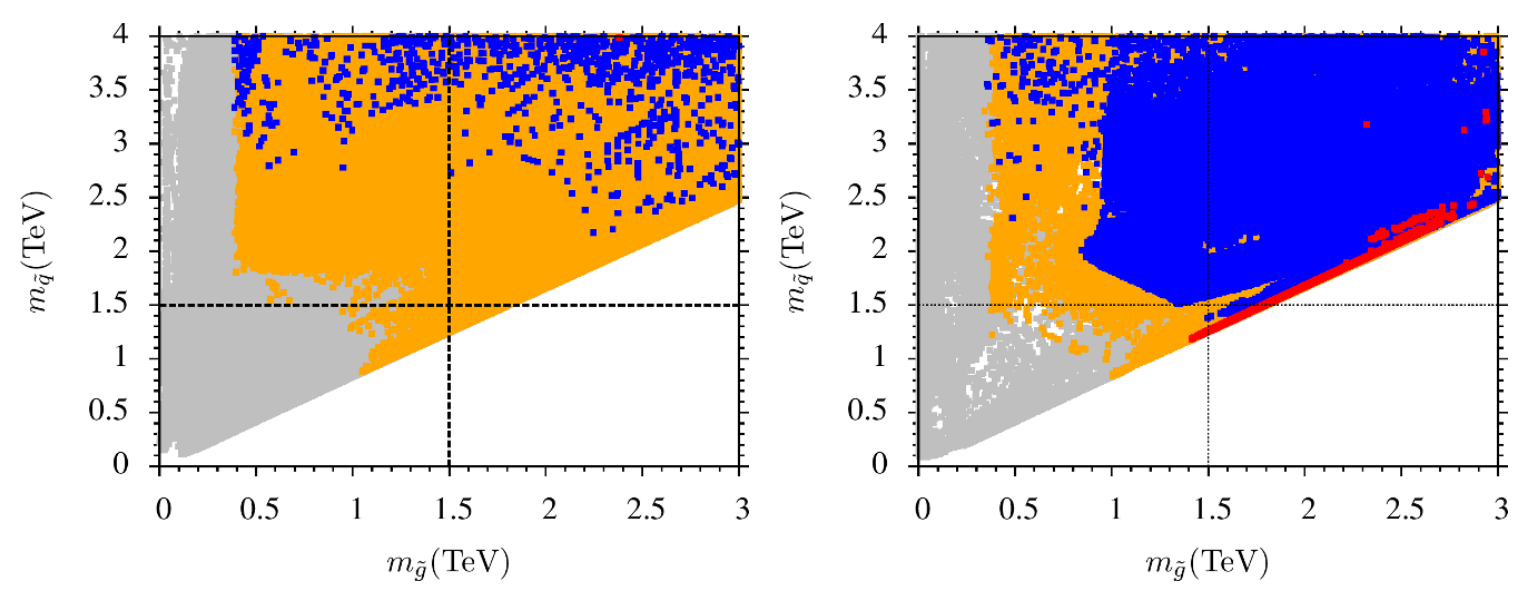

Figure 6. Plots in $m_{\tilde{g}}-m_{\tilde{q}}$ planes for CMSSM (left panel) and CMSSM-ISS (right panel). The color coding is the same as in figure 2, except that orange points do not satisfy mass bounds for gluinos and first two generation squarks, and red points are a subset of blue point solutions and also satisfy bounds for neutralino relic abundance, $0.001 \leq \Omega h^{2} \leq 1$. Dashed vertical and horizontal lines stand for current squark and gluino lower mass bounds respectively.
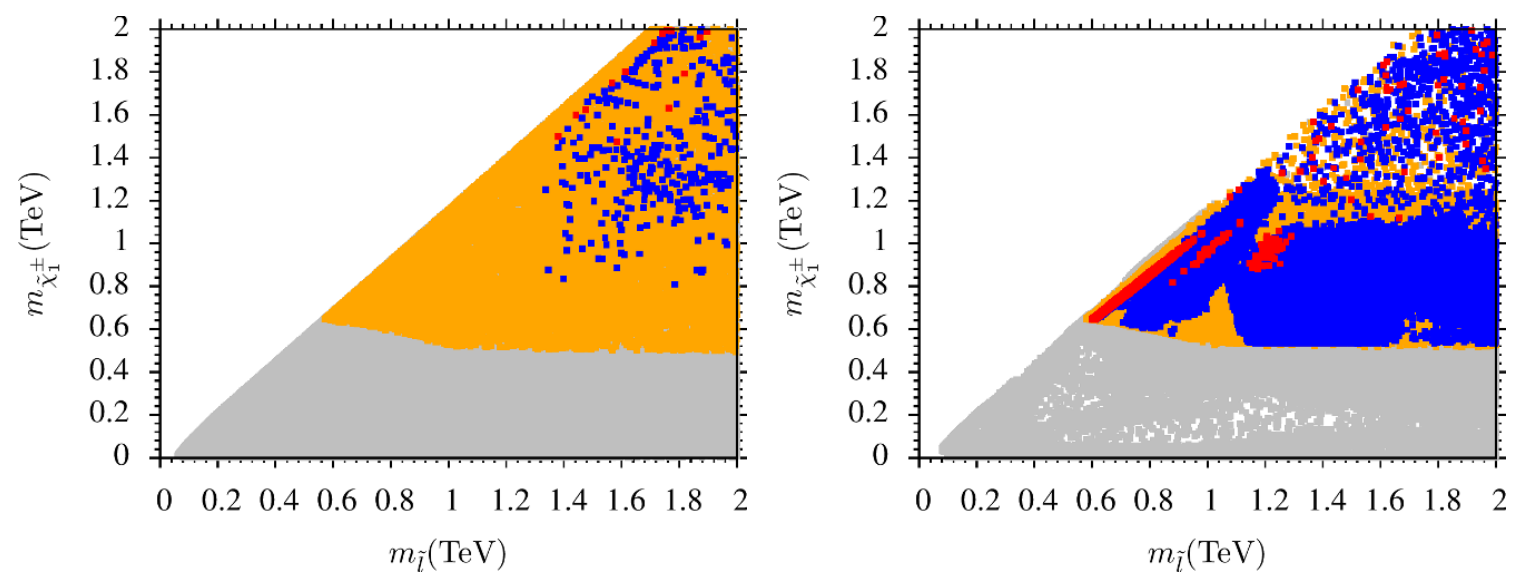

Figure 7. Plots in $m_{\tilde{l}}-m_{\tilde{\chi}_{1}^{ \pm}}$planes for CMSSM (left panel) and CMSSM-ISS (right panel). The color coding is the same as in figure 4 .

In figure 7 we display plots for $m_{\tilde{\chi}_{1}^{ \pm}}$versus $m_{\tilde{l}}$ in CMSSM (left panel) and CMSSMISS (right panel), with the color coding the same as in the previous figure. In the left panel we see from the blue points that $m_{\tilde{l}}>1.4 \mathrm{TeV}$, which may be difficult to test at the LHC. On the other hand, we see in the right panel solutions in blue and red colors around $m_{\tilde{l}} \simeq 500 \mathrm{GeV}$, which provides a glimmer of hope that sleptons employing the CMSSM-ISS mechanism may be found at the LHC.

In table 1 we display two benchmark points for the CMSSM-ISS model that are consistent with constraints in section 3. The LSP neutralino relic density in the two cases is in accord with the WMAP observations, and corresponds to stau-neutralino [75-81] (stop-neutralino [82-85]) coannihilation for point A (B). For point A, $m_{\tilde{\tau}_{1}} \approx 500 \mathrm{GeV}$, 


\begin{tabular}{|lcc|}
\hline & Point A & Point B \\
\hline$m_{0}$ & 1020.3 & 3234 \\
$M_{1 / 2}$ & 1091.1 & 684.6 \\
$A_{0} / m_{0}$ & -2.71 & -2.97 \\
$\tan \beta$ & 38 & 14.4 \\
\hline$m_{h}$ & 125 & 125 \\
$m_{H}$ & 1602 & 4744 \\
$m_{A}$ & 1592 & 4714 \\
$m_{H^{ \pm}}$ & 1604 & 4745 \\
$\mu$ & 1772 & 3727 \\
\hline$m_{\tilde{g}}$ & 2401 & 1705 \\
$m_{\tilde{\chi}_{1,2}^{0}}$ & $\mathbf{4 7 6}, 902$ & $\mathbf{3 1 2}, 608$ \\
$m_{\tilde{\chi}_{3,4}^{0}}$ & 1769,1772 & 3724,3724 \\
$m_{\tilde{\chi}_{1,2}^{ \pm}}^{ \pm}$ & 905,1773 & 614,3733 \\
\hline$m_{\tilde{u}_{L, R}}$ & 2391,2314 & 3492,3476 \\
$m_{\tilde{t}_{1,2}}$ & 1569,1983 & $\mathbf{3 4 7}, 2376$ \\
\hline$m_{\tilde{d}_{L, R}}$ & 2392,2305 & 3493,3479 \\
$m_{\tilde{b}_{1,2}}$ & 1940,2035 & 2400,3262 \\
\hline$m_{\tilde{\nu}_{1}}$ & 1248 & 3265 \\
$m_{\tilde{\nu}_{3}}$ & 792 & 2024 \\
\hline$m_{\tilde{e}_{L, R}}$ & 1252,1098 & 3261,3245 \\
$m_{\tilde{\tau}_{1,2}}$ & $\mathbf{4 9 7}, 820$ & 2040,3027 \\
\hline$\sigma_{S I}(\mathrm{pb})$ & $1.57 \times 10^{-11}$ & $1.71 \times 10^{-15}$ \\
$\sigma_{S D}(\mathrm{pb})$ & $5.05 \times 10^{-9}$ & $7.3 \times 10^{-13}$ \\
$\Omega_{h^{2}}$ & 0.114 & 0.092 \\
\hline
\end{tabular}

Table 1. Masses (in GeV units) and other parameters for two CMSSM-ISS benchmark points satisfying all phenomenological constraints discussed in section 3. Points A and B are chosen from the stau-neutralino coannihilation and the stop-neutralino coannihilation regions respectively.

$m_{\tilde{g}} \approx 2.4 \mathrm{TeV}$, the first two generation squarks are close to $2 \mathrm{TeV}$, while slepton masses are around $1-2 \mathrm{TeV}$. For point $\mathrm{B}, m_{\tilde{t}_{1}} \approx 350 \mathrm{GeV}, m_{\tilde{g}} \approx 1.7 \mathrm{TeV}$, the first two generation squark masses are about $3.4 \mathrm{TeV}$, while slepton masses are around $3.2 \mathrm{TeV}$.

\subsection{NUHM2 and inverse seesaw}

In this subsection we present the results of our scan for NUHM2 with ISS contributions (NUHM2-ISS). In figure 8 we present graphs in $m_{0}-m_{1 / 2}$ and $m_{0}-\mu$ planes, with color coding the same as in figure 2 . In the $m_{0}-m_{1 / 2}$ plane we see that the results are similar to what we found in CMSSM-ISS. Again we can have solutions compatible with all experimental constraints presented in section 3. We note that the Higgs mass constraint $123 \mathrm{GeV} \lesssim m_{h} \lesssim 127 \mathrm{GeV}$ provides the lower bounds $m_{1 / 2} \approx 500 \mathrm{GeV}$ and $m_{0} \approx 1 \mathrm{TeV}$. 

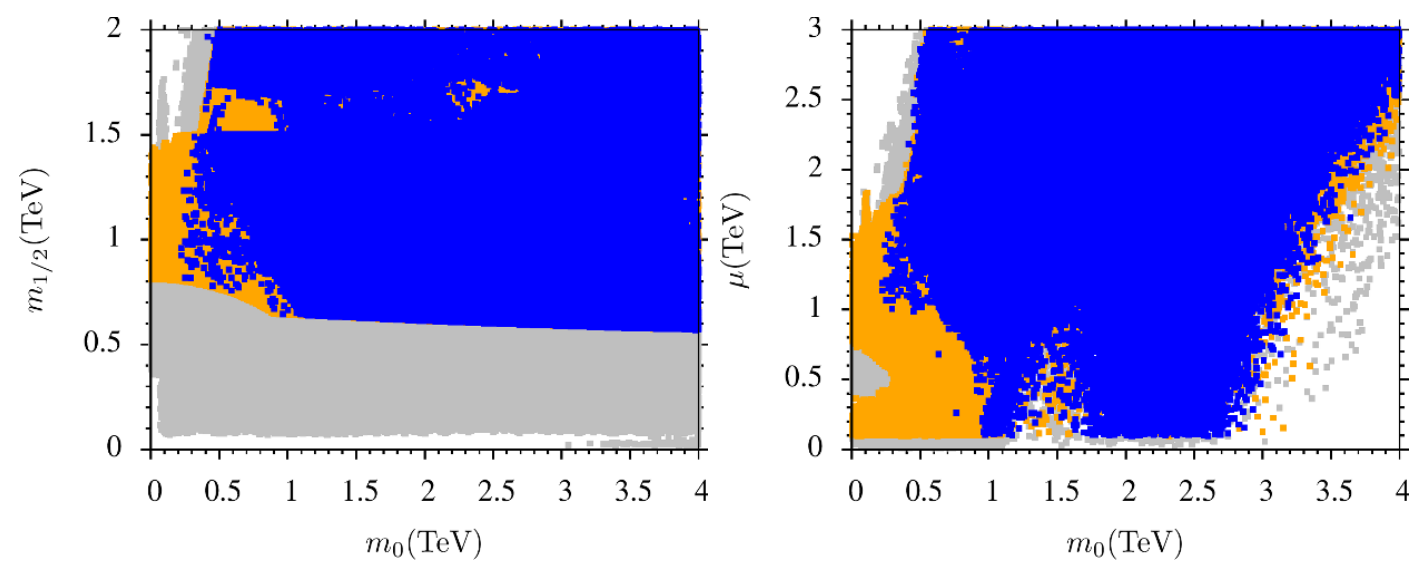

Figure 8. Plots in $m_{0}-m_{1 / 2}$ and $m_{0}-\mu$ planes for NUHM2-ISS. The color coding is the same as in figure 2 .

Since $\mu$ is a free parameter in NUHM2, we can find solutions with any value of $\mu$ compatible with the experimental data (see $m_{0}-\mu$ plot). As shown in [86, 87], a relatively small $\mu$ term is necessary, but not sufficient, to be consistent with natural supersymmetry (little hierarchy problem) criteria. We find that it is hard to fully resolve the little hierarchy problem in this scenario.

The sparticle spectrum for NUHM2-ISS is shown in figure 9, with color coding the same as in the previous figures. The top left panel shows an NLSP $\tilde{t}_{1}$ in the mass range of $220-500 \mathrm{GeV}$, which can be tested at LHC14. The top panel on right shows that the NLSP $\tilde{\tau}_{1}$ can be as light as $250 \mathrm{GeV}$, which is somewhat lighter than in the CMSSM and CMSSM-ISS scenarios. The bottom left panel shows the presence of $A$-resonance solutions. This follows from the relatively low $\mu$ values in NUHM2 (figure 8), and with $m_{H_{u}}$ and $m_{H_{d}}$ (or equivalently $\mu$ and $M_{A}$ ) being independent parameters.

In the bottom right panel we plot $m_{\tilde{\chi}_{1}^{ \pm}}$versus $m_{\tilde{\chi}_{1}^{0}}$. This graph is very different from the corresponding one for CMSSM-ISS. In NUHM2-ISS scenario, because of low $\mu$ values, the chargino can be Higgsino-like, which yields bino-Higgsino mixed dark matter. This type of solution can be seen along the solid back line. In those cases where $\mu$ is heavy, the chargino will be wino-like as in the CMSSM-ISS case. Such solutions can are displayed in the second strip in the graph. We also display a plot in the $m_{\tilde{\chi}_{1}^{0}-m_{\tilde{\nu}_{3}}}$ plane where we show a minimum value $m_{\tilde{\nu}_{3}} \approx 250 \mathrm{GeV}$, which is also consistent with the results reported in ref. [88].

In figure 10 we show graphs in $m_{\tilde{q}-} m_{\tilde{g}}$ and $m_{\tilde{\chi}_{1}^{ \pm}}-m_{\tilde{l}}$ planes. In the left panel, the orange points do not satisfy the mass bounds for gluinos and first two generation squarks. The color coding otherwise is the same as in the previous figures. Dashed vertical and horizontal lines display the current squark and gluino mass bounds.

Comparing results from figures 10 and 7, we see very small changes on the lower mass bounds for the first two generation squarks, and sleptons as well as gluinos, which is what we expected. But there are many more red points in figure 10, because in the NUHM2-ISS case, we have the additional $A$-resonance and bino-Higgsino dark matter solutions for the 

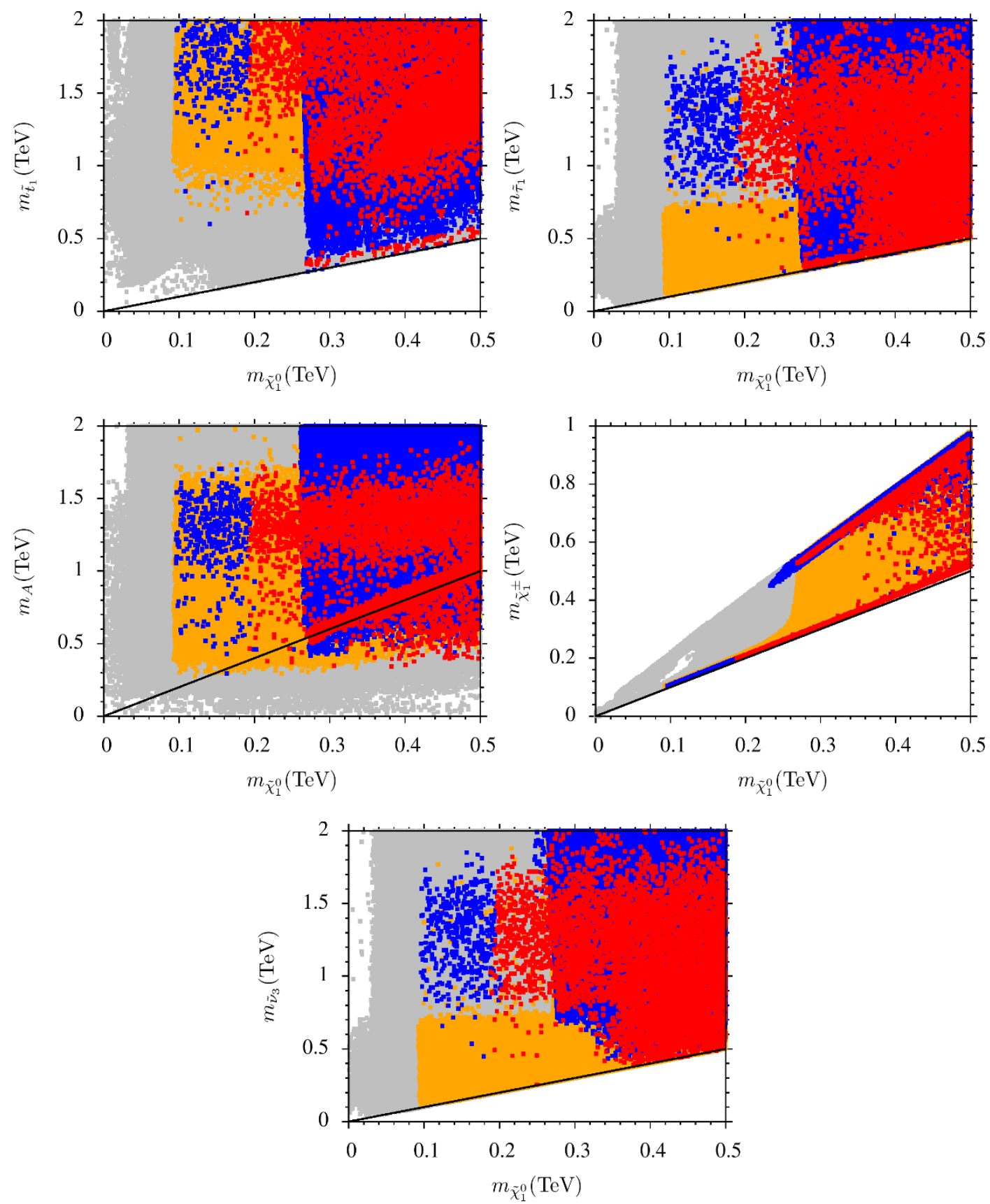

Figure 9. Plots in $m_{\tilde{\chi}_{1}^{0}}-m_{\tilde{t}_{1}}, m_{\tilde{\chi}_{1}^{0}}-m_{\tilde{\tau}_{1}}, m_{\tilde{\chi}_{1}^{0}}-m_{A}, m_{\tilde{\chi}_{1}^{0}}-m_{\tilde{\chi}_{1}^{ \pm}}$and $m_{\tilde{\chi}_{1}^{0}-} m_{\tilde{\nu}_{3}}$ planes for NUHM2-ISS. The color coding is the same as in figure 4 .

LSP neutralino relic abundance. As in the CMSSM-ISS case, we can have squarks and gluinos in a mass range which can be explored at LHC14.

In table 2 we present five benchmark points for NUHM2-ISS case which satisfy the phenomenological constraints discussed in section 3. Points 1, 2, 3, 4 and 5 are chosen, respectively, from the stau-neutralino coannihilation region, the bino-Higgsino mixed dark 

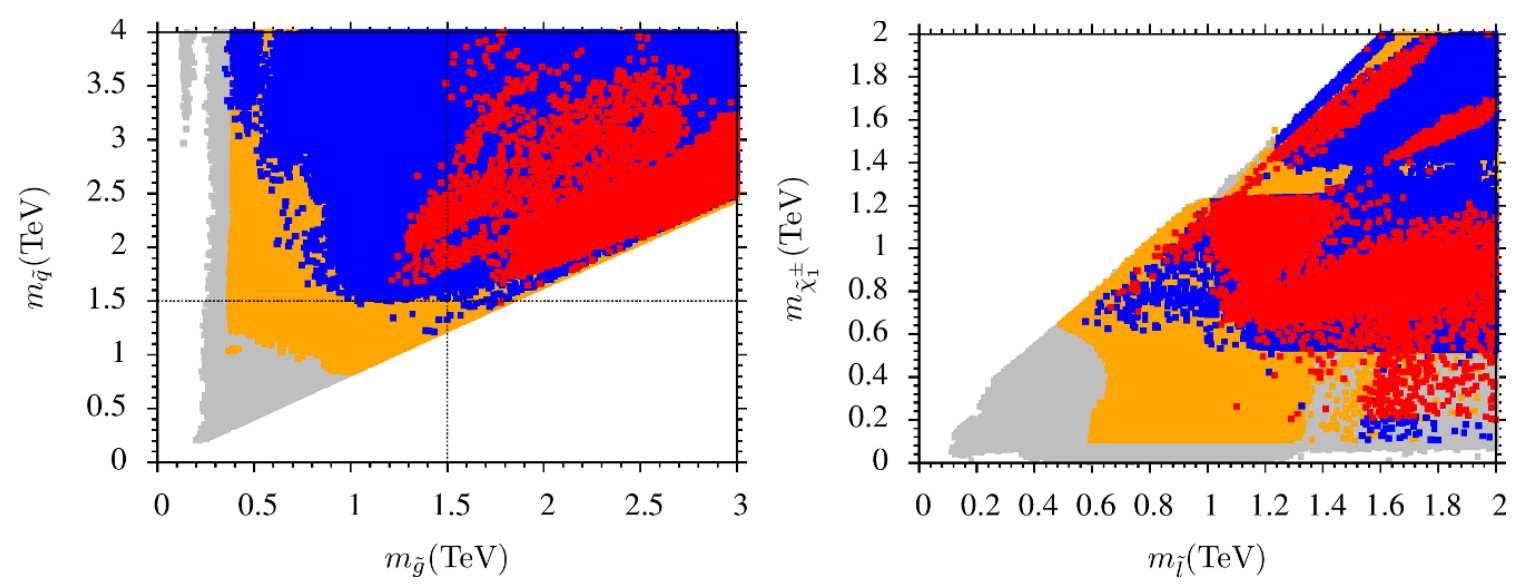

Figure 10. Plots in $m_{\tilde{g}}-m_{\tilde{q}}$ and $m_{\tilde{l}^{-}} m_{\tilde{\chi}_{1}^{ \pm}}$planes for NUHM2. In the left panel orange points do not satisfy gluino and first two generation squark mass bounds and red points are a subset of blue point solutions and also satisfy bounds for neutralino relic abundance, $0.001 \leq \Omega h^{2} \leq 1$. Dashed vertical and horizontal lines stand for current squark and gluino lower mass bounds respectively. Otherwise color coding is the same as in figure 2.

matter region, the $A$-resonance region, the sneutrino-neutralino coannihilation region, and the stop-neutralino coannihilation region. In all the five benchmark points the first two generation squarks are in the mass range $2.4-3.5 \mathrm{TeV}$, while the first two generation sleptons lie around $1.6-3 \mathrm{TeV}$. Note that for the bino-Higgsino mixed dark matter point the spin independent cross section is $6.83 \times 10^{-9} \mathrm{pb}$, which is below the current XENON100 bounds [89], but within the reach of XENON1T [90] and SuperCDMS [91].

\section{Conclusions}

The recent discovery at the LHC of a SM-like Higgs boson with mass $m_{h} \simeq 125 \mathrm{GeV}$ puts considerable stress on the MSSM. With $m_{h} \lesssim M_{Z}$ at tree level, large radiative corrections are required. Such corrections can be achieved in the MSSM either with multi-TeV stops, or with a large stop trilinear coupling and stop masses around $1 \mathrm{TeV}$. In models with universal sfermion masses at $M_{\mathrm{GUT}}$, such as CMSSM and NUHM2, this leads to heavy sleptons and 1st/2nd generation squarks which are near or beyond the ultimate LHC reach. Various MSSM extensions have been proposed to allow lighter sfermions via additional contributions to the lightest CP-even Higgs boson mass. In this paper we explored the impact of the inverse seesaw mechanism on the sparticle mass spectrum.

The ISS mechanism allows an increase of $m_{h}$ by a few $\mathrm{GeV}$, while simultaneously generating mass for neutrinos via dimension six operators. With a maximal value of the Dirac Yukawa coupling involving the up-type Higgs doublet, $m_{h}$ is increased by $2-3 \mathrm{GeV}$. As we have shown, this effect allows one to have lighter colored sparticles in CMSSM and NUHM2 scenarios which can be tested at LHC14. For example, in CMSSM-ISS the minimal value of $m_{0}$ consistent with all mass and B-physics bounds is $\sim 400 \mathrm{GeV}$, compared to CMSSM where $m_{0} \gtrsim 800 \mathrm{GeV}$. Furthermore, requiring neutralino LSP to be the cold dark 


\begin{tabular}{|lccccc|}
\hline \multicolumn{1}{c}{ Point 1} & Point 2 & Point 3 & Point 4 & Point 5 \\
\hline$m_{0}$ & 2452.3 & 1742.2 & 1573.4 & 1301.9 & 3116 \\
$M_{1 / 2}$ & 1333.4 & 1292.1 & 968.18 & 1293.1 & 857.6 \\
$A_{0} / m_{0}$ & -2.62 & -2.49 & -2.60 & -2.82 & -2.87 \\
$\tan \beta$ & 53.42 & 12.54 & 26.25 & 22.67 & 18.89 \\
$m_{H_{d}}$ & 4.5484 & 855.55 & 1.8117 & 1.7413 & 737.7 \\
$m_{H_{u}}$ & 2.0939 & 3783.2 & 2661.3 & 3060.1 & 3972 \\
\hline$m_{h}$ & 125 & 125 & 125 & 125 & 126 \\
$m_{H}$ & 1865 & 1253 & $\mathbf{8 8 2}$ & 658 & 2782 \\
$m_{A}$ & 1853 & 1245 & $\mathbf{8 7 6}$ & 654 & 2765 \\
$m_{H^{ \pm}}$ & 1867 & 1256 & 886 & 664 & 2784 \\
$\mu$ & 3483 & 6455 & 1448 & 1006 & 3149 \\
\hline$m_{\tilde{g}}$ & 2971 & 2842 & 2188 & 2816 & 2054 \\
$m_{\tilde{\chi}_{1,2}^{0}}$ & $\mathbf{6 0 0}, 1139$ & $\mathbf{5 5 6}, 656$ & $\mathbf{4 2 3 , 8 0 5}$ & $\mathbf{5 6 3 ,} 979$ & $\mathbf{3 8 8 , 7 4 8}$ \\
$m_{\tilde{\chi}_{3,4}^{0}}$ & 3447,3448 & $\mathbf{6 5 7}, 1080$ & 1445,1450 & 1015,1103 & 314,314 \\
$m_{\tilde{\chi}_{1,2}^{ \pm}}$ & 1141,3448 & 659,1070 & 807,1451 & 987,1097 & 755,3151 \\
\hline$m_{\tilde{u}_{L, R}}$ & 3565,3492 & 3052,3063 & 2479,2483 & 2836,2815 & 3515,3576 \\
$m_{\tilde{t}_{1,2}}$ & 2195,2687 & 1180,2302 & 1078,1819 & 1374,2185 & $\mathbf{4 2 8 , 2 3 0 3}$ \\
\hline$m_{\tilde{d}_{L, R}}$ & 3566,3484 & 3053,2943 & 2481,2406 & 2837,2720 & 3516,3470 \\
$m_{\tilde{b}_{1,2}}$ & 2628,2776 & 2305,2849 & 1804,2153 & 2171,2520 & 2329,3160 \\
\hline$m_{\tilde{\nu}_{1}}$ & 2605 & 2021 & 1748 & 1627 & 3225 \\
$m_{\tilde{\nu}_{3}}$ & 1503 & 804 & 818 & $\mathbf{5 6 8}$ & 1808 \\
\hline$m_{\tilde{e}_{L, R}}$ & 2606,2502 & 2022,1611 & 1749,1502 & 1630,1210 & 3222,3012 \\
$m_{\tilde{\tau}_{1,2}}$ & $\mathbf{6 2 8}, 1501$ & 824,1536 & 824,1201 & 588,972 & 1823,2693 \\
\hline$\sigma_{S I}(\mathrm{pb})$ & $1.80 \times 10^{-12}$ & $6.83 \times 10^{-9}$ & $5.11 \times 10^{-11}$ & $5.07 \times 10^{-10}$ & $2.35 \times 10^{-13}$ \\
$\sigma_{S D}(\mathrm{pb})$ & $3.80 \times 10^{-11}$ & $1.00 \times 10^{-5}$ & $2.26 \times 10^{-8}$ & $2.56 \times 10^{-7}$ & $2.1 \times 10^{-10}$ \\
$\Omega h^{2}$ & 0.108 & 0.093 & 0.113 & 0.103 & 0.122 \\
\hline & & & & & \\
\hline
\end{tabular}

Table 2. Masses (in GeV units) and ohter parameters for NUHM2-ISS benchmark points satisfying all phenomenological constraints discussed in section 3. Points 1-5 are chosen, respectively, from the stau-neutralino coannihilation, the bino-Higgsino mixed dark matter, the $A$-resonance, the sneutrino-neutralino coannihilation, and the stop-neutralino coannihilation regions.

matter pushes $m_{0}$ to $10-20 \mathrm{TeV}$ range in CMSSM, whereas in CMSSM-ISS $m_{0} \sim 400 \mathrm{GeV}$ are compatible with the CDM constraint. This means that squarks and gluinos in CMSSMISS lie within the reach of LHC14. Similarly, in NUHM2-ISS squarks and gluinos in 1.5$3 \mathrm{TeV}$ range are consistent with neutralino CDM. We have presented several LHC testable benchmark points with the desired neutralino dark matter relic abundance. 


\section{Acknowledgments}

We would like to thank Adeel Ajaib and Cem Salih Un for useful discussions. This work is supported in part by DOE Grant No. DE-FG02-12ER41808 (I.G., B.H., and Q.S.). A.M. is also supported by a DOE grant. This work used the Extreme Science and Engineering Discovery Environment (XSEDE), which is supported by the National Science Foundation grant number OCI-1053575. I.G. acknowledges support from the Rustaveli National Science Foundation under grant No. 31/98. B.H. would like to thank the Center for High Energy Physics at Peking University where part of this work was done for hospitality.

Open Access. This article is distributed under the terms of the Creative Commons Attribution License (CC-BY 4.0), which permits any use, distribution and reproduction in any medium, provided the original author(s) and source are credited.

\section{References}

[1] ATLAS collaboration, Observation of a new particle in the search for the standard model Higgs boson with the ATLAS detector at the LHC, Phys. Lett. B 716 (2012) 1 [arXiv: 1207.7214] [INSPIRE].

[2] CMS collaboration, Observation of a new boson at a mass of $125 \mathrm{GeV}$ with the CMS experiment at the LHC, Phys. Lett. B 716 (2012) 30 [arXiv:1207.7235] [INSPIRE].

[3] M.S. Carena and H.E. Haber, Higgs boson theory and phenomenology, Prog. Part. Nucl. Phys. 50 (2003) 63 [hep-ph/0208209] [inSPIRE].

[4] A. Djouadi, The anatomy of electro-weak symmetry breaking. II. The Higgs bosons in the minimal supersymmetric model, Phys. Rept. 459 (2008) 1 [hep-ph/0503173] [INSPIRE].

[5] I. Gogoladze, Q. Shafi and C.S. Un, Higgs boson mass from t-b- $\tau$ Yukawa unification, JHEP 08 (2012) 028 [arXiv:1112.2206] [INSPIRE].

[6] N. Karagiannakis, G. Lazarides and C. Pallis, Dark matter and Higgs mass in the CMSSM with Yukawa quasi-unification, J. Phys. Conf. Ser. 384 (2012) 012012 [arXiv:1201.2111] [INSPIRE].

[7] A. Anandakrishnan and S. Raby, Yukawa unification predictions with effective "Mirage" mediation, Phys. Rev. Lett. 111 (2013) 211801 [arXiv:1303.5125] [INSPIRE].

[8] M. Adeel Ajaib, I. Gogoladze, Q. Shafi and C.S. Un, A predictive Yukawa unified $\mathrm{SO}(10)$ model: Higgs and sparticle masses, JHEP 07 (2013) 139 [arXiv:1303.6964] [INSPIRE].

[9] M. Badziak, M. Olechowski and S. Pokorski, Light staus and enhanced Higgs diphoton rate with non-universal gaugino masses and $\mathrm{SO}(10)$ Yukawa unification, JHEP 10 (2013) 088 [arXiv: 1307.7999] [INSPIRE].

[10] M. Adeel Ajaib, I. Gogoladze, F. Nasir and Q. Shafi, Revisiting mGMSB in light of a 125 GeV Higgs, Phys. Lett. B 713 (2012) 462 [arXiv:1204.2856] [INSPIRE].

[11] H. Baer, V. Barger, P. Huang and A. Mustafayev, Implications of a high mass light MSSM Higgs scalar for SUSY searches at the LHC, Phys. Rev. D 84 (2011) 091701 [arXiv:1109.3197] [INSPIRE]. 
[12] H. Baer, V. Barger and A. Mustafayev, Implications of a $125 \mathrm{GeV}$ Higgs scalar for LHC SUSY and neutralino dark matter searches, Phys. Rev. D 85 (2012) 075010 [arXiv:1112.3017] [INSPIRE].

[13] A. Arbey, M. Battaglia, A. Djouadi, F. Mahmoudi and J. Quevillon, Implications of a 125 GeV Higgs for supersymmetric models, Phys. Lett. B 708 (2012) 162 [arXiv:1112.3028] [INSPIRE].

[14] M. Carena, S. Gori, N.R. Shah and C.E.M. Wagner, A $125 \mathrm{GeV}$ SM-like Higgs in the MSSM and the $\gamma \gamma$ rate, JHEP 03 (2012) 014 [arXiv:1112.3336] [INSPIRE].

[15] S. Akula, B. Altunkaynak, D. Feldman, P. Nath and G. Peim, Higgs boson mass predictions in SUGRA unification, recent $L H C-7$ results and dark matter, Phys. Rev. D 85 (2012) 075001 [arXiv:1112.3645] [InSPIRE].

[16] M. Kadastik, K. Kannike, A. Racioppi and M. Raidal, Implications of the 125 GeV Higgs boson for scalar dark matter and for the CMSSM phenomenology, JHEP 05 (2012) 061 [arXiv:1112.3647] [INSPIRE].

[17] O. Buchmüller et al., Higgs and supersymmetry, Eur. Phys. J. C 72 (2012) 2020 [arXiv:1112.3564] [INSPIRE].

[18] J. Cao, Z. Heng, D. Li and J.M. Yang, Current experimental constraints on the lightest Higgs boson mass in the constrained MSSM, Phys. Lett. B 710 (2012) 665 [arXiv:1112.4391] [INSPIRE].

[19] K.A. Olive, The impact of XENON100 and the LHC on supersymmetric dark matter, J. Phys. Conf. Ser. 384 (2012) 012010 [arXiv:1202.2324] [INSPIRE].

[20] L. Roszkowski, E.M. Sessolo and Y.-L.S. Tsai, Bayesian implications of current LHC supersymmetry and dark matter detection searches for the constrained MSSM, Phys. Rev. D 86 (2012) 095005 [arXiv: 1202.1503] [INSPIRE].

[21] A.H. Chamseddine, R.L. Arnowitt and P. Nath, Locally supersymmetric grand unification, Phys. Rev. Lett. 49 (1982) 970 [INSPIRE].

[22] R. Barbieri, S. Ferrara and C.A. Savoy, Gauge models with spontaneously broken local supersymmetry, Phys. Lett. B 119 (1982) 343 [InSPIRE].

[23] L.J. Hall, J.D. Lykken and S. Weinberg, Supergravity as the messenger of supersymmetry breaking, Phys. Rev. D 27 (1983) 2359 [INSPIRE].

[24] E. Cremmer, P. Fayet and L. Girardello, Gravity induced supersymmetry breaking and low-energy mass spectrum, Phys. Lett. B 122 (1983) 41 [INSPIRE].

[25] N. Ohta, Grand unified theories based on local supersymmetry, Prog. Theor. Phys. 70 (1983) 542 [INSPIRE].

[26] ATLAS collaboration, Search for squarks and gluinos with the ATLAS detector in final states with jets and missing transverse momentum using $4.7 \mathrm{fb}^{-1}$ of $\sqrt{s}=7 \mathrm{TeV}$ proton-proton collision data, Phys. Rev. D 87 (2013) 012008 [arXiv:1208.0949] [INSPIRE].

[27] CMS collaboration, Search for supersymmetry in hadronic final states using MT2 in pp collisions at $\sqrt{s}=7 \mathrm{TeV}$, JHEP 10 (2012) 018 [arXiv:1207.1798] [INSPIRE].

[28] CMS collaboration, CMS at the high-energy frontier. Contribution to the update of the European strategy for particle physics, CMS-NOTE-2012-006 (2012). 
[29] M.C. Gonzalez-Garcia, M. Maltoni, J. Salvado and T. Schwetz, Global fit to three neutrino mixing: critical look at present precision, JHEP 12 (2012) 123 [arXiv:1209.3023] [INSPIRE].

[30] R.N. Mohapatra et al., Theory of neutrinos: a white paper, Rept. Prog. Phys. 70 (2007) 1757 [hep-ph/0510213] [INSPIRE].

[31] R.N. Mohapatra, Mechanism for understanding small neutrino mass in superstring theories, Phys. Rev. Lett. 56 (1986) 561 [INSPIRE].

[32] R.N. Mohapatra and J.W.F. Valle, Neutrino mass and baryon number nonconservation in superstring models, Phys. Rev. D 34 (1986) 1642 [InSPIRE].

[33] I. Gogoladze, N. Okada and Q. Shafi, NMSSM and seesaw physics at LHC, Phys. Lett. B 672 (2009) 235 [arXiv:0809.0703] [INSPIRE].

[34] I. Gogoladze, B. He and Q. Shafi, Inverse seesaw in NMSSM and 126 GeV Higgs boson, Phys. Lett. B 718 (2013) 1008 [arXiv:1209.5984] [INSPIRE].

[35] J. Guo, Z. Kang, T. Li and Y. Liu, Higgs boson mass and complex sneutrino dark matter in the supersymmetric inverse seesaw models, JHEP 02 (2014) 080 [arXiv:1311.3497] [INSPIRE].

[36] J.R. Ellis, K.A. Olive and Y. Santoso, The MSSM parameter space with nonuniversal Higgs masses, Phys. Lett. B 539 (2002) 107 [hep-ph/0204192] [INSPIRE].

[37] J.R. Ellis, T. Falk, K.A. Olive and Y. Santoso, Exploration of the MSSM with nonuniversal Higgs masses, Nucl. Phys. B 652 (2003) 259 [hep-ph/0210205] [INSPIRE].

[38] H. Baer, A. Mustafayev, S. Profumo, A. Belyaev and X. Tata, Direct, indirect and collider detection of neutralino dark matter in SUSY models with non-universal Higgs masses, JHEP 07 (2005) 065 [hep-ph/0504001] [INSPIRE].

[39] P.S.B. Dev and R.N. Mohapatra, TeV scale inverse seesaw in $\mathrm{SO}(10)$ and leptonic non-unitarity effects, Phys. Rev. D 81 (2010) 013001 [arXiv:0910.3924] [INSPIRE].

[40] S. Khalil, TeV-scale gauged B - L symmetry with inverse seesaw mechanism, Phys. Rev. D 82 (2010) 077702 [arXiv: 1004.0013] [InSPIRE].

[41] Z. Kang, J. Li, T. Li, T. Liu and J. Yang, Asymmetric sneutrino dark matter in the NMSSM with minimal inverse seesaw, arXiv:1102.5644 [INSPIRE].

[42] S. Khalil, H. Okada and T. Toma, Right-handed sneutrino dark matter in supersymmetric $B-L$ model, JHEP 07 (2011) 026 [arXiv:1102.4249] [INSPIRE].

[43] F.-X. Josse-Michaux and E. Molinaro, A common framework for dark matter, leptogenesis and neutrino masses, Phys. Rev. D 84 (2011) 125021 [arXiv:1108.0482] [INSPIRE].

[44] H. An, P.S.B. Dev, Y. Cai and R.N. Mohapatra, Sneutrino dark matter in gauged inverse seesaw models for neutrinos, Phys. Rev. Lett. 108 (2012) 081806 [arXiv:1110.1366] [INSPIRE].

[45] P.S.B. Dev, S. Mondal, B. Mukhopadhyaya and S. Roy, Phenomenology of light sneutrino dark matter in cMSSM/mSUGRA with inverse seesaw, JHEP 09 (2012) 110 [arXiv:1207.6542] [INSPIRE].

[46] L. Basso, O. Fischer and J.J. van der Bij, A natural $Z^{\prime}$ model with inverse seesaw and leptonic dark matter, Phys. Rev. D 87 (2013) 035015 [arXiv:1207.3250] [INSPIRE]. 
[47] S. Banerjee, P.S.B. Dev, S. Mondal, B. Mukhopadhyaya and S. Roy, Invisible Higgs decay in a supersymmetric inverse seesaw model with light sneutrino dark matter, JHEP 10 (2013) 221 [arXiv: 1306.2143] [INSPIRE].

[48] K.S. Babu, I. Gogoladze, M.U. Rehman and Q. Shafi, Higgs boson mass, sparticle spectrum and little hierarchy problem in extended MSSM, Phys. Rev. D 78 (2008) 055017 [arXiv:0807.3055] [INSPIRE].

[49] S.P. Martin, Extra vector-like matter and the lightest Higgs scalar boson mass in low-energy supersymmetry, Phys. Rev. D 81 (2010) 035004 [arXiv:0910.2732] [INSPIRE].

[50] H. Baer, F.E. Paige, S.D. Protopopescu and X. Tata, ISAJET 7.48: a Monte Carlo event generator for $p p, \bar{p} p$, and $e^{+} e^{-}$interactions, hep-ph/0001086 [INSPIRE].

[51] Tevatron Electroweak Working Group, CDF and D0 collaborations, Combination of CDF and D0 results on the mass of the top quark, arXiv:0903.2503 [INSPIRE].

[52] Particle Data Group collaboration, J. Beringer et al., Review of particle physics, Phys. Rev. D 86 (2012) 010001 [InSPIRE].

[53] H. Baer, M. Brhlik, C.-h. Chen and X. Tata, Signals for the minimal gauge mediated supersymmetry breaking model at the Fermilab Tevatron collider, Phys. Rev. D 55 (1997) 4463 [hep-ph/9610358] [INSPIRE].

[54] H. Baer, M. Brhlik, D. Castano and X. Tata, $b \rightarrow s \gamma$ constraints on the minimal supergravity model with large $\tan \beta$, Phys. Rev. D 58 (1998) 015007 [hep-ph/9712305] [INSPIRE].

[55] D. Eriksson, F. Mahmoudi and O. Stal, Charged Higgs bosons in minimal supersymmetry: updated constraints and experimental prospects, JHEP 11 (2008) 035 [arXiv:0808.3551] [INSPIRE].

[56] CMS collaboration, Observation of a new boson with mass near $125 \mathrm{GeV}$ in pp collisions at $\sqrt{s}=7$ and $8 \mathrm{TeV}$, JHEP 06 (2013) 081 [arXiv:1303.4571] [INSPIRE].

[57] LHCb collaboration, First evidence for the decay $B_{s}^{0} \rightarrow \mu^{+} \mu^{-}$, Phys. Rev. Lett. 110 (2013) 021801 [arXiv:1211.2674] [INSPIRE].

[58] Heavy Flavor Averaging Group collaboration, Y. Amhis et al., Averages of b-hadron, c-hadron and $\tau$-lepton properties as of early 2012, arXiv:1207.1158 [INSPIRE].

[59] Heavy Flavor Averaging Group collaboration, D. Asner et al., Averages of b-hadron, c-hadron and $\tau$-lepton properties, arXiv:1010.1589 [INSPIRE].

[60] WMAP collaboration, G. Hinshaw et al., Nine-year Wilkinson Microwave Anisotropy Probe (WMAP) observations: cosmological parameter results, Astrophys. J. Suppl. 208 (2013) 19 [arXiv: 1212.5226] [INSPIRE].

[61] J.L. Feng, K.T. Matchev and T. Moroi, Multi-TeV scalars are natural in minimal supergravity, Phys. Rev. Lett. 84 (2000) 2322 [hep-ph/9908309] [INSPIRE].

[62] J.L. Feng, K.T. Matchev and T. Moroi, Focus points and naturalness in supersymmetry, Phys. Rev. D 61 (2000) 075005 [hep-ph/9909334] [InSPIRE].

[63] J.L. Feng, K.T. Matchev and F. Wilczek, Neutralino dark matter in focus point supersymmetry, Phys. Lett. B 482 (2000) 388 [hep-ph/0004043] [INSPIRE].

[64] K.L. Chan, U. Chattopadhyay and P. Nath, Naturalness, weak scale supersymmetry and the prospect for the observation of supersymmetry at the Tevatron and at the CERN LHC, Phys. Rev. D 58 (1998) 096004 [hep-ph/9710473] [INSPIRE]. 
[65] H. Baer, I. Gogoladze, A. Mustafayev, S. Raza and Q. Shafi, Sparticle mass spectra from SU(5) SUSY GUT models with b - $\tau$ Yukawa coupling unification, JHEP 03 (2012) 047 [arXiv: 1201.4412] [INSPIRE].

[66] I. Gogoladze, S. Raza and Q. Shafi, Light stop from $b-\tau$ Yukawa unification, Phys. Lett. B 706 (2012) 345 [arXiv:1104.3566] [InSPIRE].

[67] M. Adeel Ajaib, T. Li and Q. Shafi, Stop-neutralino coannihilation in the light of LHC, Phys. Rev. D 85 (2012) 055021 [arXiv:1111.4467] [inSPIRE].

[68] B. He, T. Li and Q. Shafi, Impact of LHC searches on NLSP top squark and gluino mass, JHEP 05 (2012) 148 [arXiv:1112.4461] [INSPIRE].

[69] M. Drees and M.M. Nojiri, The neutralino relic density in minimal $N=1$ supergravity, Phys. Rev. D 47 (1993) 376 [hep-ph/9207234] [InSPIRE].

[70] H. Baer and M. Brhlik, Cosmological relic density from minimal supergravity with implications for collider physics, Phys. Rev. D 53 (1996) 597 [hep-ph/9508321] [InSPIRE].

[71] H. Baer and M. Brhlik, Neutralino dark matter in minimal supergravity: direct detection versus collider searches, Phys. Rev. D 57 (1998) 567 [hep-ph/9706509] [InSPIRE].

[72] H. Baer et al., Yukawa unified supersymmetric $\mathrm{SO}(10)$ model: cosmology, rare decays and collider searches, Phys. Rev. D 63 (2000) 015007 [hep-ph/0005027] [INSPIRE].

[73] A.B. Lahanas, D.V. Nanopoulos and V.C. Spanos, Neutralino dark matter elastic scattering in a flat and accelerating universe, Mod. Phys. Lett. A 16 (2001) 1229 [hep-ph/0009065] [INSPIRE].

[74] A.B. Lahanas and V.C. Spanos, Implications of the pseudoscalar Higgs boson in determining the neutralino dark matter, Eur. Phys. J. C 23 (2002) 185 [hep-ph/0106345] [INSPIRE].

[75] J.R. Ellis, T. Falk and K.A. Olive, Neutralino-stau coannihilation and the cosmological upper limit on the mass of the lightest supersymmetric particle, Phys. Lett. B 444 (1998) 367 [hep-ph/9810360] [INSPIRE].

[76] J.R. Ellis, T. Falk, K.A. Olive and M. Srednicki, Calculations of neutralino-stau coannihilation channels and the cosmologically relevant region of MSSM parameter space, Astropart. Phys. 13 (2000) 181 [Erratum ibid. 15 (2001) 413] [hep-ph/9905481] [INSPIRE].

[77] R.L. Arnowitt, B. Dutta and Y. Santoso, Coannihilation effects in supergravity and D-brane models, Nucl. Phys. B 606 (2001) 59 [hep-ph/0102181] [INSPIRE].

[78] M.E. Gómez, G. Lazarides and C. Pallis, Supersymmetric cold dark matter with Yukawa unification, Phys. Rev. D 61 (2000) 123512 [hep-ph/9907261] [INSPIRE].

[79] M.E. Gómez, G. Lazarides and C. Pallis, Yukawa unification, $b \rightarrow s \gamma$ and bino-stau coannihilation, Phys. Lett. B 487 (2000) 313 [hep-ph/0004028] [INSPIRE].

[80] M.E. Gómez, G. Lazarides and C. Pallis, Yukawa quasi-unification, Nucl. Phys. B 638 (2002) 165 [hep-ph/0203131] [INSPIRE].

[81] T. Nihei, L. Roszkowski and R. Ruiz de Austri, Exact cross-sections for the neutralino slepton coannihilation, JHEP 07 (2002) 024 [hep-ph/0206266] [INSPIRE].

[82] C. Boehm, A. Djouadi and M. Drees, Light scalar top quarks and supersymmetric dark matter, Phys. Rev. D 62 (2000) 035012 [hep-ph/9911496] [INSPIRE].

[83] J.R. Ellis, K.A. Olive and Y. Santoso, Calculations of neutralino stop coannihilation in the CMSSM, Astropart. Phys. 18 (2003) 395 [hep-ph/0112113] [InSPIRE]. 
[84] J. Edsjö, M. Schelke, P. Ullio and P. Gondolo, Accurate relic densities with neutralino, chargino and sfermion coannihilations in mSUGRA, JCAP 04 (2003) 001 [hep-ph/0301106] [INSPIRE].

[85] J.L. Diaz-Cruz, J.R. Ellis, K.A. Olive and Y. Santoso, On the feasibility of a stop NLSP in gravitino dark matter scenarios, JHEP 05 (2007) 003 [hep-ph/0701229] [INSPIRE].

[86] H. Baer et al., Post-LHC7 fine-tuning in the mSUGRA/CMSSM model with a $125 \mathrm{GeV}$ Higgs boson, Phys. Rev. D 87 (2013) 035017 [arXiv:1210.3019] [INSPIRE].

[87] I. Gogoladze, F. Nasir and Q. Shafi, Non-universal gaugino masses and natural supersymmetry, Int. J. Mod. Phys. A 28 (2013) 1350046 [arXiv:1212.2593] [INSPIRE].

[88] N. Okada, S. Raza and Q. Shafi, Particle spectroscopy of supersymmetric SU(5) in light of $125 \mathrm{GeV}$ Higgs and muon $g-2$ data, arXiv: 1307.0461 [INSPIRE].

[89] XENON100 collaboration, E. Aprile et al., Dark matter results from 225 live days of XENON100 data, Phys. Rev. Lett. 109 (2012) 181301 [arXiv:1207.5988] [INSPIRE].

[90] The XENON Dark Matter Project, http://xenon.astro.columbia.edu/XENON100_Experiment/; http://xenon.astro.columbia.edu/talks/aprile_ucla_dm2010.pdf.

[91] CDMS-II collaboration, P.L. Brink et al., Beyond the CDMS-II dark matter search: SuperCDMS, eConf C 041213 (2004) 2529 [astro-ph/0503583] [INSPIRE]. 